\title{
Effects of atmospheric electric field on muon intensity observed in GRAPES-3 experiment
}

\author{
B. Hariharan $\mid a b$, S.R. Dugad ${ }^{a b}$, S.K. Gupta ${ }^{a b}$, Y. Hayashi ${ }^{a c}$, P. Jagadeesan ${ }^{a b}$, A. Jain ${ }^{a b}$, \\ S. Kawakami ${ }^{a c}$, P.K. Mohanty ${ }^{a b}$, P.K. Nayak ${ }^{a b}$, B.S. Rao ${ }^{a b}$ \\ ${ }^{a}$ The GRAPES-3 Experiment, Cosmic Ray Laboratory, Raj Bhavan, Ooty 643001, India \\ ${ }^{b}$ Tata Institute of Fundamental Research, Mumbai 400005, India \\ ${ }^{c}$ Graduate School of Science, Osaka City University, Osaka 558-8585, Japan \\ E-mail: 89hariharanegmail.com
}

\begin{abstract}
During bad weather conditions, muon flux above $1 \mathrm{GeV}$ observed in GRAPES-3 muon telescope shows large variations. The atmospheric electric field is believed to be responsible for acceleration of cosmic ray muons. A thunderstorm event observed on 30 September 2015 is discussed here. The field of view of large area $\left(560 \mathrm{~m}^{2}\right)$ tracking muon telescope at GRAPES-3 allows study of muon intensity variations in 169 directions with $\sim 4^{\circ}$ resolution. This event shows large decrease in North-East directions, with a maximum decrease of $-0.6 \%$ in all directions combined and $-4.5 \%$ in a single direction. Muons from selected directions were combined and processed with fast Fourier transform (FFT) to remove slow and ultra-fast variations $(\triangle \mathrm{f}=10-200$ cycles per day). The filtered data shows maximum decrease of $-1.2 \%$ with statistical significance of $9 \sigma$. Monte Carlo simulations were carried out with modified CORSIKA package to incorporate electric field. Simulations show that electric potential $\sim 1 \mathrm{GV}$ is needed to get variation of $1 \%$ in GRAPES-3 muon flux.
\end{abstract}

35th International Cosmic Ray Conference - ICRC2017

10-20 July, 2017

Bexco, Busan, Korea

* Speaker. 


\section{Introduction}

The propagation of cosmic ray (CR) particles can be affected by the atmospheric electric field (AEF). The acceleration and deceleration of these particles depend on polarity of the field. The atmospheric acceleration of CR particles was first suggested by C.T.R. Wilson in 1925 [1,2]. In his hypothesis, he suggested that AEF can accelerate electrons in the atmosphere. Several ground based experiments have confirmed the influence of AEF on secondary particles in EAS. A clear linear and quadratic dependence of electric field in soft and hard components was observed by Baksan experiment [3-6]. Variations in muon flux and neutron monitor data was observed at Mt. Norikura by ICRR group [7]. Variations in soft gamma ray flux was observed by EASTOP experiment [8]. These reports did not provide directional information about the thunderstorm events. However, in GRAPES-3, the thunderstorm events can be studied with directional information in detail as discussed in following sections.

\section{The GRAPES-3 tracking muon telescope}

The muon telescope of the GRAPES-3 experiment at Ooty, India is collecting data uninterruptedly since 2001 [9]. It is designed to study high energy CR astrophysics. The primary objective of the muon telescope is to differentiate primary gamma rays from charged CR in GRAPES-3 to obtain cosmic ray composition and to probe various solar phenomena with high precision [10].

The muon telescope uses proportional counter (PRC) as the basic element. Each PRC is made of mild steel tube of $600 \mathrm{~cm}$ long, $10 \mathrm{~cm} \times 10 \mathrm{~cm}$ cross section with a wall thickness of $2.3 \mathrm{~mm}$. A muon telescope module consists of 232 PRCs arranged in 4 layers, with alternate layers placed in mutually orthogonal directions which gives sensitive area of $35 \mathrm{~m}^{2}$. The total area covered by all 16 module is $560 \mathrm{~m}^{2}$ with coverage of $2.3 \mathrm{sr}$. Two successive layers of PRCs are separated by 15 $\mathrm{cm}$ thick concrete which permits a two dimensional reconstruction of muon tracks in two vertical, orthogonal planes (Fig. 1a). The vertical separation of two layers of PRCs in the same plane is 50

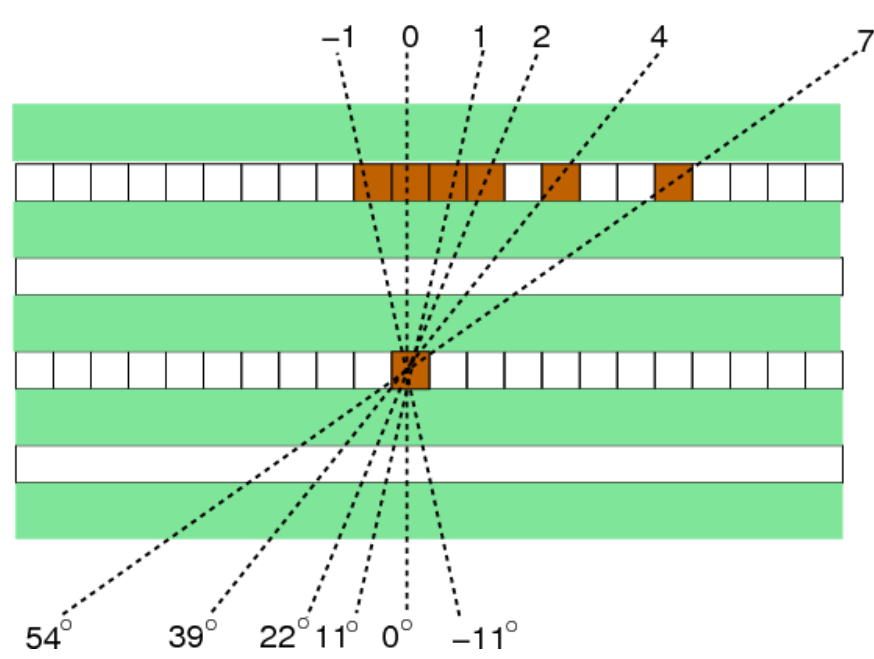

(a) Angle reconstruction in one projection

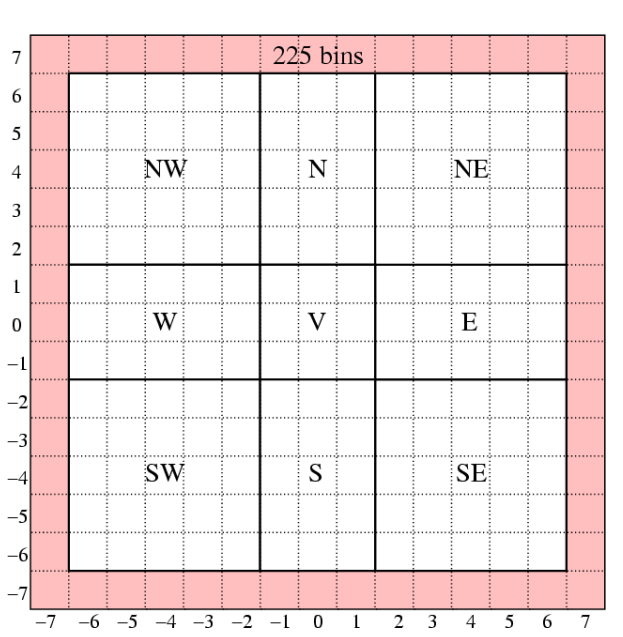

(b) Telescopic view of 225 directions

Figure 1: The GRAPES-3 muon angle system 
$\mathrm{cm}$ which allows the muon tracks to be reconstructed in 225 directions (Fig. 1b) with an accuracy of $\sim 4^{\circ}$ in the projected plane. The outer most bins are overflow bins which can be ignored and the muons from inner 169 directions can be used for physics study. To achieve an energy threshold of $1 \mathrm{GeV}$ for vertical muons, a total thickness of $\sim 550 \mathrm{~g} \mathrm{~cm}^{-2}$ in the form of concrete blocks of $2.4 \mathrm{~m}$ thickness is used as absorber. The concrete blocks have been arranged in the shape of an inverted pyramid to achieve an energy threshold of $\sec (\theta) \mathrm{GeV}$ for muons incident at a zenith angle $\theta$ with coverage up to $45^{\circ}$.

The GRAPES-3 experiment records $4 \times 10^{9}$ muons daily with statistical error of $\sim 0.002 \%$. The muon data has been successfully corrected for efficiency variation [11]. The high quality data recorded by the GRAPES-3 muon telescope allows us to study any transient effects with high precision.

\section{Electric field measurement}

The GRAPES-3 has installed, Boltek make EFM-100 atmospheric Electric Field Monitor (EFM) installed at four different locations. The EFM 'G3' is placed at the location of the muon telescope. The other EFMs namely 'GS', 'G2' and 'GL' are placed with the separation of few kilometers as shown in Fig. 2. It records AEF at every 50 millisecond(ms). On a clear weather, the observed electric field is $\sim 0.01-0.07 \mathrm{kV} \mathrm{m}^{-1}$, whereas during thunderstorm the magnitude of electric field can reach beyond $10 \mathrm{kV} \mathrm{m}^{-1}$. During lightning strokes, it was observed that the rise time of electric field discharge is comparable to the resolution of the instrument which means the discharge is much faster than $50 \mathrm{~ms}$. This instrument provides approximate distance of the lightening discharge without any directional information.

On an average, GRAPES-3 observes $\sim 30$ thunderstorm events every year. A simple and larger variation event which was observed on 30 September 2015 was selected for our study.

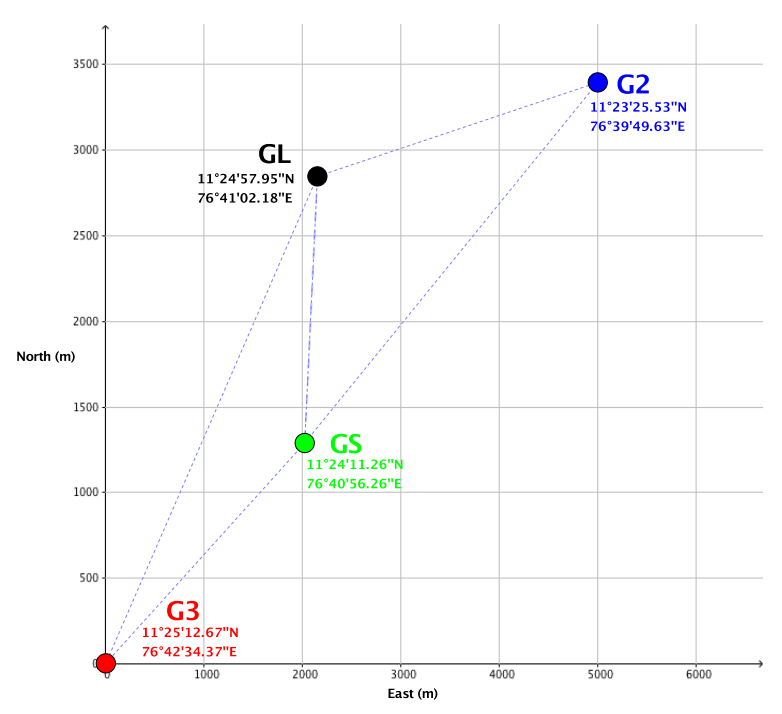

Figure 2: The GRAPES-3 atmospheric Electric Field Monitors 


\section{The event on 30 September 2015}

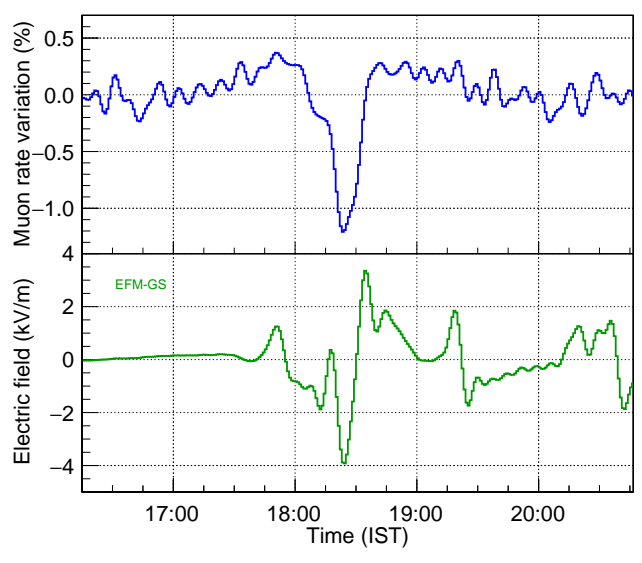

(a) Muon rate from selected directions

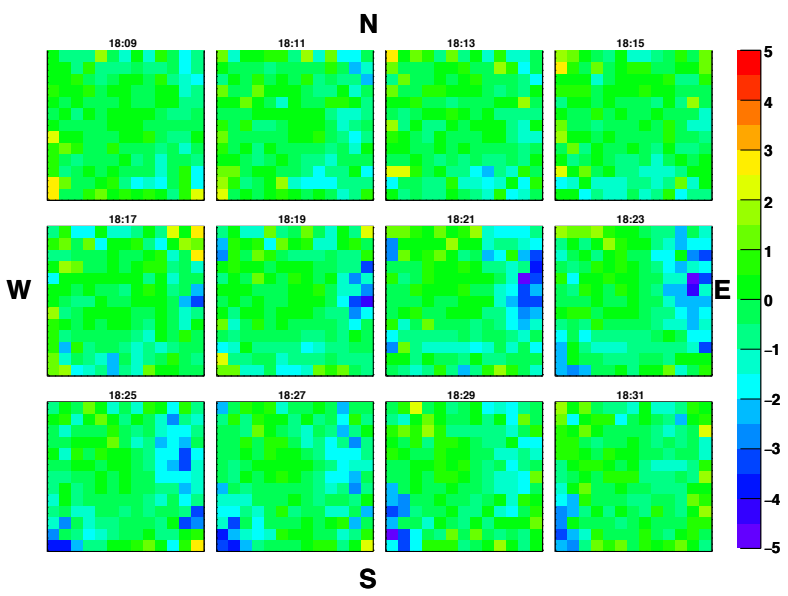

(b) Time evolution in 169 direction ( 2 min. interval)

Figure 3: Thunderstorm event on 30 September 2015

On 30 September 2015, a large variation event was observed in muon rate which showed variations in $\sim 36 \%$ of GRAPES-3 field of view. By applying quality cuts, the muon rates from 11 telescopes were combined in 1 minute bin interval to minimize statistical error. The data was corrected for atmospheric pressure variations. The combined muon rates from all directions show variation of $-0.6 \%$. Variation of $-5 \%$ were also observed in a few single directions in 169-direction. To maximize signal to background ratio, directions with muon rate variation larger than $-0.5 \%$ were selected and combined. This resulted in selection of 61 directions out of 169 directions mostly distributed in North-East region. Both the muon rate and EFM data were processed with FFT to retain frequencies from 10 to 200 cycles per day by using a sine edge rectangular band pass filter. The filtered muon rate (Fig. 3a) shows a maximum variation of $-1.2 \%(9 \sigma)$. In the event time span (Fig. 3b), one can see clear movement of muon rate variation from North-East to South-West direction which indicates the movement of electric field in thunderclouds.

The muon rate seems to have better agreement with EFM data in most of the places. However, the initial perturbations in EFM does not have correlation with muon rate. This could be due to the omni-directional measurements of the EFM instrument where as the muon rates are directional.

\section{CORSIKA simulations}

CORSIKA is a package to carry out MC simulation of EAS development [12]. It has $\sim 80000$ lines of code developed in FORTRAN and a few optional $\mathrm{C}$ routines. It can be used with various hadronic event generators like DPMJET [13], EPOS LHC [14], NEXUS [15], QGSJET 01C [16], QGSJETII-04 [17], SIBYLL 2.1 [18] and VENUS 4.12 [19] for high energy interactions and GHEISHA [20], FLUKA [21] and UrQMD [22] for low energy interactions. It is used to simulate primaries in the energy range of $10^{9} \mathrm{eV}-10^{20} \mathrm{eV}$. The secondary particles are tracked through the atmosphere until they reach observational level or up to a threshold energy defined by the user. At a given observational level, the position, momentum and time-of-flight of secondary particles are 
recorded.

The effects of electric field can be simulated with an inbuilt option 'EFIELD' in CORSIKA [23]. But the EFIELD allows simulations only for electrons and positrons. However, the GRAPES3 simulations are primarily on muons, the effects can not be studied with the existing EFIELD. So, the EFIELD option was modified to study the electric field effect on muons [24]. A total of $7.4 \times 10^{10}$ proton showers were simulated using CORSIKA v74001 with the combination of SIBYLL and FLUKA generators in the energy range of $10 \mathrm{GeV}-10 \mathrm{TeV}$. The zenith angle range was $0^{\circ} \leq \theta \leq 60^{\circ}$ and azimuthal angle range was $0^{\circ} \leq \phi \leq 360^{\circ}$. The energy threshold of secondary particles were set to $50 \mathrm{MeV}$ for hadrons, $10 \mathrm{MeV}$ for muons, $1 \mathrm{GeV}$ for electrons and $1 \mathrm{GeV}$ for gammas. The primaries were selected based on their directional cutoff rigidity through another modification in CORSIKA [25].

The simulations were carried out for 60 steps of electric field ranging from -15 to $15 \mathrm{kV} \mathrm{cm}^{-1}$ with step size of $0.5 \mathrm{kV} \mathrm{cm}^{-1}$. And a set was simulated without electric field for background. The field was uniformly applied at $6 \mathrm{~km}$ above Ooty with the cloud thickness of $2 \mathrm{~km}$. From simulations, muons above $1 \mathrm{GeV}$ observed at Ooty altitude were selected for detector simulation. The in-house developed detector simulation code was used to track CORSIKA muons for various trigger conditions and to get the angular distribution of muons in 169 directions.

\section{Results and discussions}

The simulation profile (Fig. 4a) shows decrease in muon intensity irrespective of the applied electric field. Initially, a small positive excess can be seen at negative fields. The variation in muon intensity has non-linearity from -6 to $6 \mathrm{kV} \mathrm{cm}{ }^{-1}$. A change in slope is also observed beyond \pm 6 $\mathrm{kV} \mathrm{cm}{ }^{-1}$ which leads to a rapid decrease in muon intensity. The overall profile has a quadratic dependence of electric field which agrees with earlier reports [3,4]. These results are contradictory with the expectations that the decrease and increase in different polarity of the fields are due to

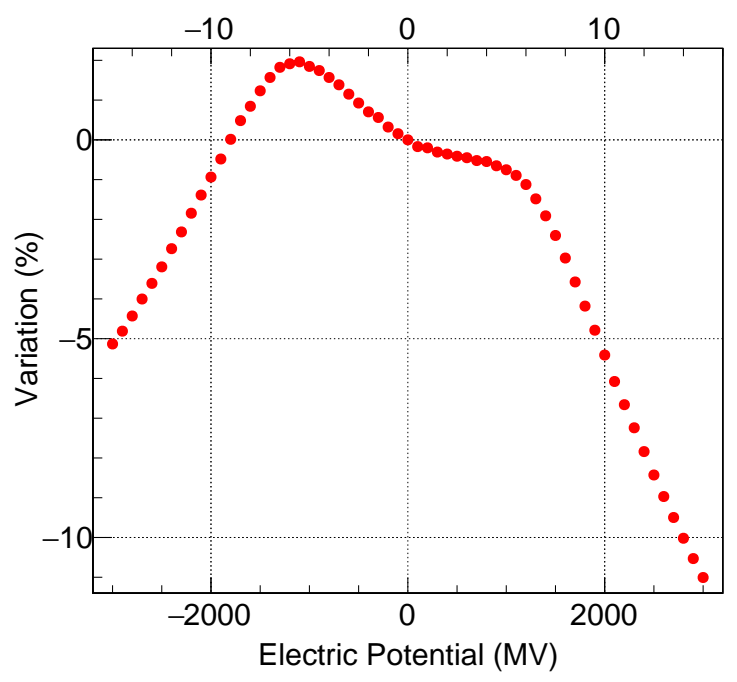

(a) Variation of muon intensity

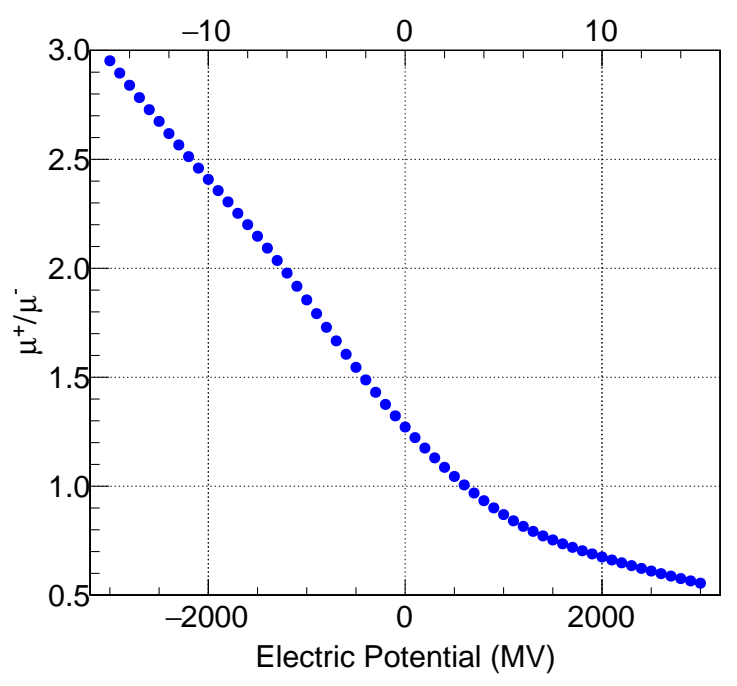

(b) Variation of muon charge ratio $\left(\mu^{+} / \mu^{-}\right)$

Figure 4: Electric field simulations 


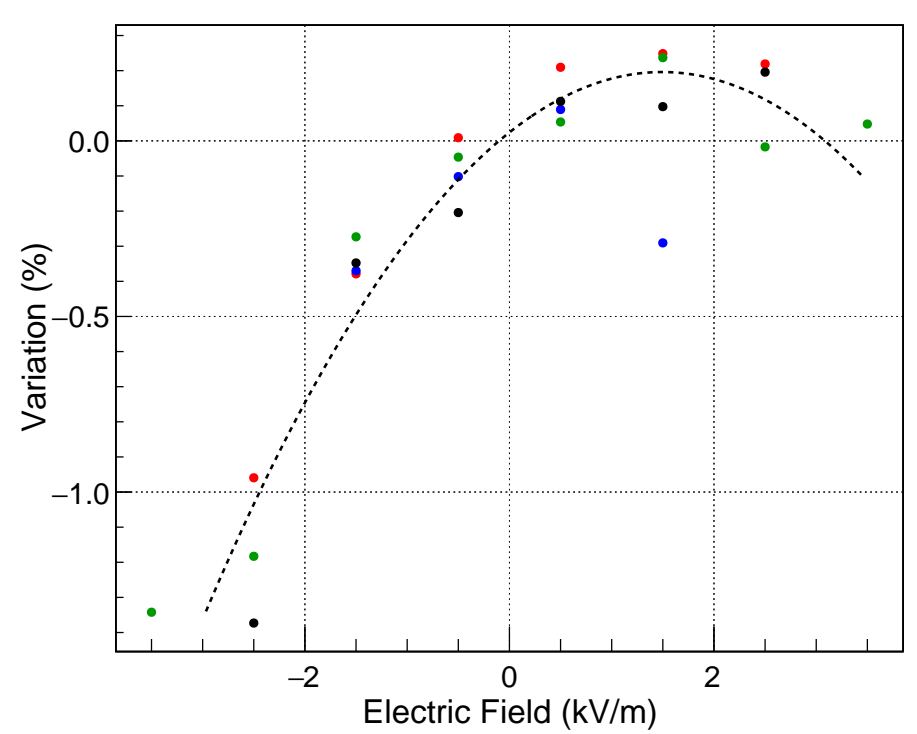

Figure 5: Measured muon rate variations of EFMs (G3, GS, G2, GL)

muon charge asymmetry. At Ooty, the muon charge ratio $\left(\mu^{+} / \mu^{-}\right)$was estimated to be $\sim 1.25$ from simulations (Fig. 4b). The amount of excess positive muons could explain the dependence of decreasing and increasing muon intensity. The change in slope which leads to a rapid fall of muon intensity is due to the change in muon decay probability beyond the mean energy of muons at Ooty. The electric potential of order $\sim 1 \mathrm{GV}$ is required to produce $\sim 1 \%$ variation in GRAPES-3 muon flux.

The dependence of muon rate and electric field was obtained from observation (Fig. 5). It shows similar quadratic dependence as seen in the simulation which was also reported by other experiments $[5,6]$.

\section{Acknowledgement}

We thank D.B. Arjunan, G.P. Francis, V. Jeyakumar, S. Kingston, K. Manjunath, S. Murugapandian, S. Pandurangan, B. Rajesh, K. Ramadass, V. Santoshkumar, M.S. Shareef, C. Shobana, R. Sureshkumar and other colleagues for their help in running and maintenance of the GRAPES-3 tracking muon telescope.

\section{References}

[1] C.T.R. Wilson, Proc. Camb. Phil. Soc. 1925, Vol. 22 534-8

[2] C.T.R. Wilson, Proc. Phys. Soc. Lond. 1925, 2 Vol. 37 32D-37D

[3] V.V. Alexeenko, A.E. Chudakov, V.G. Sborshikov, and V.A. Tizengauzen, in Proceedings of the 19th International Conference on Cosmic Rays (National Aeronautics and Space Administration, Greenbelt, 1985), Vol. 5, p. 352. 
[4] V.V. Alexeenko et al., in Proceedings of the 20th International Conference on Cosmic Rays (Nauka, Moscow, 1987), Vol. 4, p. 272

[5] V.V. Alexeenko, N.S. Khaerdinov, A.S. Lidvansky, and V.B. Petkov, in Proceedings of the 27th International Conference on Cosmic Rays (Copernicus Gesellschaft, Hamburg, 2001), Vol. 10, p. 4161 .

[6] V.V. Alexeenko et al, Phys.Lett. 301A, 299 (2002).

[7] Y.Muraki et al., Phys. Rev. D 69, 123010 (2004).

[8] M. Aglietta et al., in Proceedings of the 26th International Conference on Cosmic Rays (The University of Utah, SaltLake City, 1999), Vol. 7, p. 351.

[9] Y. Hayashi et al., Nucl. Instrum. and Methods A 545 (2005) 643-657.

[10] S.K. Gupta et al., Nucl. Instrum. Methods A 540 (2005) 311.

[11] P.K. Mohanty et al., Proceedings of Science PoS(ICRC2017)357

[12] D. Heck, J. Knapp, J.N. Capdevielle, G. Schatz, and T. Thouw, Report FZKA 6019 (1998), Forschungszentrum Karlsruhe; available from http://www-ik.fzk.de/corsika/physics_description/corsika_phys.html

[13] J. Ranft, Phys. Rev. D51 (1995) 64; arXiv:hep-ph/9911213 and hep-ph/9911232 (1999)

[14] T. Pierog et al., arXiv:1306.0121[hep-ph] (2013)

[15] H.J. Drescher, M. Hladik, S. Ostapchenko, T. Pierog, and K. Werner, Phys. Rep. 350 (2001) 93 (preprint hep-ph/0007198 (2000))

[16] N.N. Kalmykov, S.S. Ostapchenko, and A.I. Pavlov, Nucl. Phys. B (Proc. Suppl.) 52B (1997) 17

[17] S.S. Ostapchenko, Phys. Rev. D83 (2011) 014018

[18] R. Engel, T.K. Gaisser, P. Lipari, and T. Stanev, Proc. $26^{\text {th }}$ Int. Cosmic Ray Conf., Salt Lake City (USA), 1 (1999) 415; E.-J. Ahn, R. Engel, T.K. Gaisser, P. Lipari, and T. Stanev, Phys. Rev. D80 (2009) 094003

[19] K. Werner, Phys. Rep. 232 (1993) 87

[20] H. Fesefeldt, Report PITHA-85/02 (1985), RWTH Aachen; available from: http://cds.cern.ch/record/162911/files/CM-P00055931.pdf

[21] A. Fassò, A. Ferrari, S. Roesler, P.R. Sala, G. Battistoni, F. Cerutti, E. Gadioli, M.V. Garzelli, F. Ballarini, O.Ottolenghi, A. Empl and J. Ranft, The physics models of FLUKA: status and recent developments, Computing in High Energy and Nuclear Physics 2003 Conference (CHEP2003), La Jolla, CA (USA), March 24-28, 2003 (paper MOMT005); eConf C0303241 (2003); arXiv:hep-ph/0306267; http://www.fluka.org/references.html

[22] S.A. Bass et al., Prog. Part. Nucl. Phys. 41 (1998) 225; M. Bleicher et al., J. Phys. G: Nucl. Part. Phys. 25 (1999) 1859;

http://urqmd.org/

[23] D. Heck et al., DOI(KIT): 10.5445/IR/1000028191

http://publikationen.bibliothek.kit.edu/1000028191

[24] B. Hariharan et al., Proceedings of Science PoS(ICRC2017)305.

[25] B. Hariharan et al., Proceedings of Science PoS(ICRC2015)448. 